\title{
Prevalence and Molecular Characterization of Carbapenem resistance Gram negative bacilli among hospitalized patients in Khartoum state
}

Hana Salaheldin Elbadawi ( $\sim$ hanasalah200@gmail.com )

Institute of Endemic Diseases https://orcid.org/0000-0003-2192-7932

Kamal Elhag

Soba University Hospital

Elsheikh Mahgoub

University of Khartoum Faculty of Medicine

Hisham N Altayb

Sudan University of Science and Technology

Muzamil Mahdi Abdel Hamid

Institute of Endemic Diseases, University of Khartoum

Research article

Keywords: Gram negative bacilli, carbapenemase producing, carbapenem resistance gene

Posted Date: September 7th, 2019

DOI: https://doi.org/10.21203/rs.2.14050/v1

License: (c) (1) This work is licensed under a Creative Commons Attribution 4.0 International License.

Read Full License 


\section{Abstract}

\section{Background}

Carbapenems are broad-spectrum $\beta$-lactam antibiotics widely prescribed for the treatment of multidrugresistant gram negative bacilli in systemic infections. In the past ten years the Carbapenem resistance among gram-negative bacilli is rapidly expanding across nosocomial infection isolates. This study was conducted to determine the prevalence and to characterize Carbapenem resistance genes among Gram negative bacilli (GNB) isolated from patients treated in hospitals in Khartoum state, Sudan.

\section{Methods}

A cross-sectional laboratory based study was conducted over six months at the microbiology department in Soba University Hospital and Institute of Endemic Diseases, University of Khartoum. A total of 206 GNB isolates from different clinical specimens were analyzed for carbapenem resistance genes using phenotypic tests and affirmed by genes detection. Multiplex PCR was performed for each strain to detect the carbapenemase genes, including those encoding the NDM-, VIM-, and IMP -type Metallo-betalactamases, the class A KPC-type carbapenemases, and the class D OXA-48 enzyme. In addition to CTXM, TEM and SHV. DNA sequencing and bioinformatics analysis were used to detect genes subtypes.

Results

Of 206 isolates, 171 (83\%) positive phenotypically and 121 (70.7\%) from 171 isolates were confirmed for present one or more carbapenemase gene. NDM-types were the most predominant genes, blaNDM 107(88.4\%), followed by blaIMP 7 (5.7\%), blaOXA-48 5(4.1\%), blaVIM 2 (1.6\%) and blaKPC 0 (0\%). Coresistance genes with NDM producing Gram-negative bacilli were detected in 87 (81.3\%) of all NDM positive isolates. Correlation between phenotypic and genotypic resistance was observed ( $\mathrm{P}$-value < 0.001). Carbapenemase genes were mostly detected in the K. pneumoniae $70(42.6 \%)$, followed by P. aeruginosa $33(20 \%)$, A. baumannii $30(18.2 \%)$ and E. coli with 18 strains (10.9\%). NDM-1 was detected in 75 isolates (70\%), other subtypes of NDM were identified by sequencing were NDM- 5 and NDM-6.

\section{Conclusions}

The prevalence of carbapenemase producing bacilli was fond to be high in Khartoum hospitals. NDM was found to be the most prevalent carbapenemase genes among clinical isolates and belong to Indian lineage. For prevention infection control and regular surveillance must be enhanced.

\section{Introduction}

Carbapenems are important broad-spectrum $\beta$-lactam antibiotics widely prescribed for the curing of multidrug-resistant gram negative bacilli in systemic infections. Carbapenems have been considered as a robust antibiotic to treat the extended spectrum $\beta$-lactamases (ESBLs) in the past ten years [1]. ESBLs are one of the most common resistant genes distributed among gram negative bacilli through plasmids and 
transposons [2] and the novel $\beta$-lactamases with direct carbapenem-hydrolyzing activity has contributed to an increased prevalence of carbapenem resistant Enterobacteriaceae (CRE), which is causing therapeutic failure worldwide [3]. Carbapenemase enzymes including New Delhi Metallo-beta-lactamase (blaNDM), veron integron metallo-beta-lactamases (blaVIM), imipenemase (blalMP), Klebsiella pneumoniae carbapenemases (blaKPC), and oxacillinase-48 (blaOXA-48) [4]. These enzymes are encoded by what is termed carbapenem resistance determining genes (CRDG), which hydrolyze $\beta$-lactam drugs including Carbapenems and other $\beta$-lactam agents [5]. Moreover resistant to carbapenem can occur by other mechanisms including overproduction of ESBL or AmpC enzyme in combination with porin mutations by reduced outer membrane permeability and activation of multidrug efflux pumps in response to antibiotic exposure [6]. Carbapenem resistance genes are enhancing mechanism of antibiotic resistance among the family Enterobacteriaceae and non-lactose fermenting gram-negative bacilliin consequence of the selective pressure assessed by inadequate use of carbapenem and third generation cephalosporins [7]. Moreover plasmids coding for carbapenemase enzymes may carry co-resistance genes for other $\beta$-lactam and non $\beta$-lactam antibiotics [5]. Detection of carbapenemase production by clinical microbiology laboratory is essential to guide the clinicians to provide appropriate therapy and update treatment guild lines furthermore provide evidence on clinically observed treatment failure using molecular analysis.

The major problem in Sudan is the lack of control over antimicrobial use and despite the lack of specific data about the incidence of AMR in Sudan. This study was designed to describe the current situation of carbapenemase producing Gram-negative bacteria and determine the resistance genes involved in Khartoum state, Sudan.

\section{Material And Methods}

\section{Study design and clinical strains}

A cross-sectional laboratory based study was conducted at the microbiology department in Soba University Hospital and institute of Endemic Diseases, University of Khartoum; involving gram negative clinical bacterial isolates, that suspected as carbapenemase producing strains based on carbapenem sensitivity testing zone inhibition (zone size less than $20 \mathrm{~mm}$ ). These were isolated from a variety of clinical specimens: blood, urine, swabs, sputum, a tip of catheters, and body fluids, between October 2016 and February 2017 from hospitalized patients in Soba University Hospital. Quality control strains used in antimicrobial susceptibility testing and the biochemical test had been used for primary identification [8]. Molecular identification (PCR) using species specific primers for Klebsiella pneumoniae, Escherichia coli, Pseudomonas aeruginosa, Acinetobacter baumannii and Universal primers (16SrRNA) were used for more confirmation Table.1. All these strains stored in $20 \%$ glycerol at $-20^{\circ} \mathrm{C}$ until use.

\section{Subculturing and Disk Diffusion Susceptibility Testing}


Isolates were subcultured on blood agar (BA) and then subjected to susceptibility testing to the following antibiotics: Amoxycillin clavulanate (AMC) $(30 \mu \mathrm{g})$; Cefuroxime (CXM) $(30 \mu \mathrm{g})$; Cephalexin (CL) $(30 \mu \mathrm{g})$; Ceftriaxone (CRO) $(30 \mu \mathrm{g})$; Ceftazidime (CAZ) $(30 \mu \mathrm{g})$; Cefotaxime (CTX) $(30 \mu \mathrm{g})$; Meropenem (MEM) $(10 \mu \mathrm{g})$

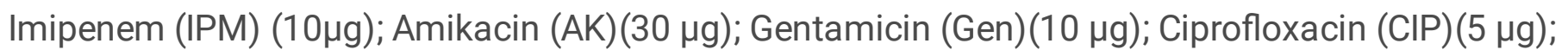
Trimethoprim-sulfamethoxazole (SXT) $(25 \mu \mathrm{g})$; Temocillin (TEM)(30 C); Azteroname (AZT)(30 $\mu \mathrm{g})$. That was done using the Kirby Bauer disk diffusion; each isolate was swabbed on the Muller-Hinton agar and the antibiotic discs were placed on top and incubated at $37 \mathrm{C}$ for 18-24 hours and interpreted according to Clinical and Laboratory Standards Institute (CLSI) guidelines [9].

\section{Phenotypic screening and confirmatory test for carbapenemase}

The bacterial isolates screened for carbapenemase production according to CLSI guidelines (CLSI, 2017). In this method, carbapenem antibiotics, meropenem (MEM) and imipenem (IPM) discs (10 g, each) (Mast Diagnostic, UK) were used and Isolates that showed a zone of inhibition $\leq 21 \mathrm{~mm}$ in diameter for meropenem were considered as suspected carbapenemase producers. Phenotypic confirmatory test for carbapenemases production by Boronic acid synergy test for class A $\beta$-lactamases, the EDTA synergy for Metallo- $\beta$-lactamase and the Modified Hodges Test (MHT) for detecting KPC and OXA-48 producers [10].

\section{Detection of Carbapenemase encoding genes}

The PCR was carried out using thermal cycler (analytikjena ${ }^{\circledR}$ Biometra TADVANCED, Germany), by using the following primers (Macrogen, Korea), bla VIM, bla IMP, bla NDM, bla NDM-1, bla KPC, bla OXA-48, blaTEM, bla SHV and bla CTX-M genes Table 2. The reaction was carried out in a total reaction volume of $25 \mu \mathrm{l}(5 \mu \mathrm{l}$ Master mix of Maxime RT premix kit (iNtRON BIOTECHNOLOGY, Seongnam, Korea), $0.6 \mu \mathrm{l}$ of forward primer, $0.6 \mu \mathrm{l}$ of reverse primer, $2 \mu \mathrm{l}$ of DNA and $16.8 \mu \mathrm{l}$ deionized sterile water). The reaction was conducted for 30 cycles [6]. The purity and integrity of each PCR product were evaluated electrophoresis in a $2 \%$ agarose gel in TBE $1 \mathrm{X}$, that contain $2.5 \mu \mathrm{l}$ of $(20 \mathrm{mg} / \mathrm{ml})$ ethidium bromide at $100 \mathrm{~V}$ for $40 \mathrm{~min}$. The specific amplified product was detected by comparing with 100 base-pairs standard DNA ladder (iNtRON BIOTECHNOLOGY, Seongnam, Korea) Bands were visualized under U.V transilluminater (analytikjena® Biometra BDAcompact, Germany).

\section{DNA Sequencing}

The PCR product of bla NDM genes and 16srRNA were purified and Sanger sequencing was performed by Macrogen Company (Seoul, Korea)..

\section{Bioinformatics Analysis}


First of all we ensure the ambiguous sites are correctly called and determined the overall quality of the sequences proofed the nucleotides chromatogram by using Finch TV software version 1.4.0 (http://www.geospiza.com/Products/finchtv.shtml). Then nucleotides sequences of the NDM genes achieved were searched for sequence similarity using nucleotide BLAST [11] (http:

//blast.ncbi.nlm.nih.gov/Blast.cgi). Multiple sequence alignment for highly similar sequences, which retrieved from NCBI using the MEGA version 7 software [12]. Phylogenetic tree of bla NDM genes and their evolutionary relationship with those obtained from database were conducted using MEGA version 7 [12].

\section{Statistical analysis}

Data were analyzed using SPSS software version 20.0. Cross tabulation was used to present the relations between data, qualitative data were performed through $\chi 2$ test, and significance was set at $p \leq 0.05$.

\section{Results}

\section{Antimicrobial susceptibility}

Antibiotic resistance pattern is shown in (figure 1). Out of 206 isolates tested by disk diffusion test, the highest percentage of resistance $98 \%, 93.5 \%$ were found in ampicillin and cephalexin respectively, followed by amoxicillin clavulanic acid $90 \%$, cefotaxime $89.7 \%$, ceftriaxone $88.4 \%$, ceftazidime $84.2 \%$ and aztreonam $66 \%$, temocillin $64 \%$, co-trimoxazole $78.4 \%$ and nitrofurantoin $75.2 \%$. Resistance rate also higher in ciprofloxacin $83.1 \%$, gentamicin $85 \%$ and amikacin $70 \%$. Meropenem and imipenem were the most effective antibiotic tested and resistant were observed in high rates with $63.1 \%$ and $61.6 \%$ respectively.

\section{Prevalence of carbapenemase producing gram-negative bacilli based on phenotypic tests}

Carbapenemase activity was detected in 171 (83\%) of the 206 clinical isolates were positive for the production of one or more carbapenemase enzymes by phenotypic methods as the following $24(14 \%)$ by MHT method and Boronic acid screen, 105 (61.5\%) by the EDTA test and 42 (24.5\%) of the isolates were positive for both EDTA and Boronic acid methods. Details of the carbapenemase activity among different strains by phenotypic tests are shown in Table 3. That mean the MBL type is the most prevalent type of carbapenemase hydrolysis enzyme among Gram-negative bacilli in Khartoum state, OXA and KPC types are present at a low level.

\section{Prevalence and distribution of Carbapenemase genes among gram negative bacilli}


Carbapenemase genes were detected in 121 (70.7\%) from 171 positive carbapenemase producing isolates using PCR, one or more carbapenemase genes were detected in the strains. blaNDM was detected in the highest rate among the isolates mainly in K. pneumonia, which was the species with the highest number of these genes. blaNDM was also detected higher in other strains $A$. baumannii, $P$. aeruginosa and E. coli. The most prevalent gene was blaNDM 107(88.4\%), followed by blalMP7 (5.7\%), blaOXA-48 5(4.1\%), blaVIM 2 (1.6\%) and blaKPC 0 (0\%). And ESBL were detected among these strains, it showed a high prevalence in 183 isolates (88.8\%) as the following CTXM 127(61.6\%), SHV 84(40.7\%) and TEM 80(38.8). The genes were unevenly distributed among the different study isolates for more details table 4.

\section{Co resistance genes carried with NDM gene among gram-negative bacilli}

Many strains were herbed more than one gene with NDM gene. Co-resistance carbapenemase genes were observed in few isolates, NDM + OXA-48 were detected in three strains, while NDM+ VIM and NDM+IMP were detected in two strains one for each other. On the other hand, ESBL were observed in high prevalence with NDM in $87(81.3 \%)$ of NDM positive isolates (107). Most of the strains carried NDM with one ESBL gene in (43.5\%) as the following; NDM+ CTXM in (24 isolates, 27.6\%), NDM+ TEM (8 isolates, $9.1 \%$ ), and NDM+ SHV (6 isolates, 6.8\%). Strains carried NDM with two ESBL genes in (39.2\%) as the following:NDM+ CTXM+ SHV10 isolates, $11.5 \%), N D M+C T X M+$ TEM10 isolates, $11.5 \%), N D M+S H V+$ TEM14 isolates, $16.2 \%)$. Strains carried NDM with three ESBL genes, NDM+ CTXM+ SHV +TEM (15 isolates, $17.3 \%$ ). The destitution of co resistance genes among different gram negative bacilli is shown in table 5.

\section{The frequency of carbapenemase producer Gram-negative bacilli by type of specimens and hospital units}

Carbapenemase producers were more frequent distributed among clinical isolates from blood (36\%) and then followed by wounds (24\%), urine (21\%), body fluids (7\%) and catheter tips and sputum (6\%) for both.

With regard to the distribution of Carbapenemase producer among hospital units, the most carbapenemase producer isolated from neonatal intensive care unit NICU 32(26\%), followed by medicine wards 26(22\%) and pediatric wards 22 (18\%), Surgery 18(15\%), ICU 15(12\%) and Renal Unit 8(7\%).

\section{Molecular characterization of NDM genes}

Out of 107 NDM genes detected 75 (70\%) were NDM-1, other subtypes of NDM were identified by sequencing including NDM-5, and NDM-6. Figure 2 


\section{Bioinformatics analysis of NDM genes}

Fourteen samples were successfully sequenced by Macrogen Company. All sample showed 97-100\% similarity with NDM genes from the NCBI database with accession number MF379688 and MG764089.

\section{Multiple sequence alignment}

The Nucleotide sequences of NDM Deoxyribonucleic acid (DNA) sequences were compared against DNA databank using BLASTp. Fourteen different NDM beta-lactamases genes were compared against the NDM in the database, (http://blast.ncbi.nlm.nih.gov/Blast.cgi), they have produced a significant alignment to NDM-1 beta-lactamase of Klebsiella pneumoniae (gb/MK425054l), and the strains were shown $97 \%$ to $100 \%$ identity.

Multiple sequence alignment of NDM proteins done by MEGA7 software version 7.0.9.0 against similar proteins that obtained from BLASTp, NDM-1 from Sudan were similar to IKX100583.1/ Escherichia coli NDM-1 (blaNDM) gene from India and / MH891562/ Klebsiella pneumoniae NDM-1 from Bangladesh. NDM-5 from Sudan were similar to / MH991817 /, Escherichia coli NDM-5fromIndia and / MH168510/ Klebsiella pneumoniae NDM-5 from Bangladesh while NDM-6 from Sudan were similar to / MH683607 / Escherichia coli from India, / JN967644 / Escherichia coli from the United States and / JQ235754 / Escherichia coli from New Zealand.

\section{Nucleotide sequence accession number}

The sequence of the 14 NDM genes have been deposited in the GenBank database under accession numbers MK033562, MK033563, MK033564, MK363705, MK363706, MK363707, MK363708, MK363709, MK363710, MK371542, MK371543, MK371544, MK37154 5, and MK371546.

\section{Phylogenetic tree}

The phylogenetic analysis of the NDM proteins sequences revealed that the NDM-1 and NDM-5 were related to the same NDM lineage as the Indian and Bangladeshi isolates. The NDM-6 gene was found as closed to NDM-6 from India, New Zealand, and the United States as shown in Figure 2.

\section{Discussion}

Carbapenems have become the drugs of choice for the treatment of severe nosocomial infections caused by Gram-negative bacilli; however, carbapenemase producing Gram-negative bacilli have been reported worldwide. CRE is a considerable health problem worldwide and associated with increased mortality. The rapid detection of carbapenem resistance and adequate treatment of such cases is therefore mandatory. This study was therefore undertaken, to determine the prevalence of different types of carbapenemase 
producing bacteria among Gram-negative bacilli isolated from various hospitalized patients in Khartoum State. Accurate detection of carbapenemase producing microorganisms is a challenge for the laboratories, requiring not only phenotypic tests but also genotypic tests for all genes associated with carbapenemase production. In the present study, among 206 isolates 171(83\%) were positive by phenotypic analysis including strains with resistance to carbapenem. Furthermore, genotypic analysis detected 121 (70.7\%) positive strains. This finding indicates that the studied resistance is not only associated with enzyme encoding genes but also due to other resistance mechanisms such as overproduction of ESBLs, porin loss or mutations [13,14].

The current situation according to this study, show that the prevalence of carbapenemase producing among different gram negative isolates is increasing up to (83\%). This finding is higher than the incidence in a previous study conducted in Khartoum state in 2017 which showed the prevalence was $56 \%$ by phenotypic tests [15] and other done in 2013 by Ali reported the MBL was 37.7\% among Pseudomonas spp. isolates in Khartoum state [16]. This high frequency of MBL in Khartoum state is a result of excessive use of third generation cephalosporins, in addition to selective of ESBL with prevalence $88 \%$, make the treatment option of those patients was meropenem and excessive use of it lead to release of carbapenem resistance genes. This finding agrees with a study in Egypt reported carbapenem resistance rate was $62.7 \%$ among Enterobacteriaceae [17]. As well, carbapenem resistance has been observed in Africa in high rate study conducted by Okoche et a., in 2015 in Uganda. He found $28.6 \%$ of strains were carbapenemase producer [18]. In Tanzania the prevalence of carbapenemase producer was 35\% [19] and higher incidence 68\% In South Africa [20]. Low prevalence was observed in Nigeria 11.9\% [21]. This finding in the poor populations in Africa may be as a result of unrestricted use of antibiotics in these countries where most people consume the antibiotics without prescription by a clinician [22].

Carbapenemase genes have been recognized during the past ten years, and these genes are associated with mobile genetic elements that allow their rapid circulation among bacterial strains, for instance, NDM type have a potential for rapid spread within the country and to other countries [23]. In this study, carbapenemase genes were detected by using PCR in 121 (70.7\%) of the resistant isolates. The most prevalent gene among the isolates was blaNDM (88.4\%) mainly in K. pneumonia and other gram negative bacilli including $A$. baumannii, P. aeruginosa and E. coli, this agrees with studies in India reported the NDM gene was observed between $31 \%$ and 55\% of Carbapenemase resistance Enterobacteriaceae [24,25], and study in South Africa published the most carbapenemase gene was NDM among $K$. pneumonia (20). As well, NDM-1 was reported as the most common carbapenemase gene in Saudi Arabia and other Middle Eastern countries [26].

Carbapenemase genes are reported to be more frequent in some regions. For example KPC genes are dominant in some countries such as Greece, Israel, and USA, while NDM genes are prevalent in isolates reported from the Far East, India, and Pakistan [14]. Carbapenemase production in Turkey mostly occurs in OXA type genes (23). OXA-48 was reported first from Turkey, subsequently followed by reports from Middle Asia and Europe as well [27]. In this current study the genes were unevenly distributed among the 
different study isolates. In comparison with other carbapenem resistant genes were detected in low prevalence comparing with NDM gene blaIMP (5.7\%), blaOXA-48 (4.1\%), blaVIM (1.6\%) and blaKPC (0\%). This finding disagrees with many studies, in Okoche study, the most common gene was blaVIM 1(0.7\%), and blaNDM-1 (2.6\%) was the lowest gene [18], while Mushi reported IMP types were the most predominant at (21.6\%) in his study (19). Other studies reported blaOXA-48 was the most prevalence gene $[15,28]$. In our study KPC wasn't detected among the isolates that disagree with international reports of high prevalence of KPC genes [14,29].

The blaNDM-1 was first identified in a clinical isolate of K. pneumoniae in New Delhi, India, and suddenly got disseminated around the world [30]. NDM variants have been described, differing by several amino acid changes. A first variant, NDM-2, has been described in an A. baumannii clinical isolate from Egyptian patient in Germany, NDM-4, NDM-5, NDM-6 have been detected from E. coli in India and NDM-7 from E.coli in France [30]. In this study, 107 NDM producer strains had been identified using PCR, the most subtype 75 (70\%) were NDM-10ther subtypes of NDM were detected by sequencing including NDM- 5, and NDM- 6 among different Gram negative bacilli including K. pneumoniae, E. coli, A. baumannii, $P$. aeruginosa and Enterobacter spp.

Carbapenemase genes are becoming largely distributed among Enterobacteriaceae, A. baumannii, $P$. aeruginosa and other Gram-negative bacilli. The prevalence of carbapenemase producer in each species in this study, higher frequency in A. baumannii (37.3 \%) followed by K. pneumoniae (27.3\%), P. aeruginosa (24.8\%) and E. coli (21.1\%), which agree with many studies that reported A. baumannii and $K$. pneumonia were the most predominant carbapenemase producer strains [31,32]. The prevalence of carbapenemase producer varies from area to area. This variation could be attributed to differences in time of collection of isolates and differences in study populations and designs. A study in Turkey showed the most carbapenemase strains were K. pneumoniae (13.6\%), Pseudomonas spp. (17.8\%), A. baumannii (13.8\%), S. maltophilia 7.5\% and E. coli 2.8\% [33]. In Nigeria the highest prevalence of carbapenemase producers was in P. mirabilis (16.0\%), then P. aeruginosa, K. pneumoniae (13.3\% each) and E. coli (11.5\%) [21], while in Tanzania, E. coli was the most prevalent species with carbapenemase producing (14\%), followed by K. pneumoniae (10.57\%), P. aeruginosa (10.13\%), K. oxytoca (1.76\%) and A. baumannii (1.3\%) [19].

Carbapenemase-encoding genes had been commonly associated with bacteria isolated from blood, urine, wound swabs, and sputum as reported in many studies in Uganda [19], Tanzania [34], Nigeria [21], and India [35]. In this study Carbapenem producer were more frequently isolated from blood (39\%) followed by wound (25\%) and urine (22\%) this is in line with study in South Africa which reported blood was the most common specimen type (25\%), followed by urine (22\%) [20].

Many studies considered young age as a risk factor for CRE infection which agrees with current finding, that carbapenemase-producing Gram negative bacilli were most frequent in neonate age group isolated from nursery and pediatric wards (26\% and $18 \%$ respectively). Besides, carbapenemase producers were 
observed in high rate among elderly patients from medicine (22\%) and ICU (12\%), which agrees with another study that found that CRE to be more frequently isolated in the elderly [36].

Carbapenem resistance gram negative bacilli are usually resistant to other routinely used antimicrobial agents [37-39]. The Plasmids carrying carbapenemase genes like NDM-1 are diverse and can harbor a high number of additional resistance genes (e.g., ESBL-alleles) as well as other carbapenemase genes like Oxa-48 types, VIM types, and so forth, as the source of multidrug resistance in one single bacterium $[25,40]$. Moreover mechanisms of resistance to $\beta$-lactam by producing ESBL, AmpC and carbapenemase were also noticed as some of the isolates produce different combinations of the enzymes. In our study co-resistance of NDM with OXA-48, VIM and IMP were reported in few strains. Co-resistance with ESBL (CTXM, SHV and TEM) was detected in high prevalence 87/107 (81.3\%) of NDM positive isolates. Most of the strains carried NDM with one ESBL gene in (43.5\%), NDM with two ESBL genes in (39.2\%) and NDM with three ESBL genes in (17.3\%). That with harmony with various studies reported co-resistance among clinical strains [41,42]. These co-production genes among some isolates as observed in this study are indicative of the existence of multi-drug resistant bacteria pathogens. That may be responsible for treatment failure and outbreaks of infections caused by resistant organisms. Resulting in more hospitalization and higher treatment costs as well as disease complications [43].

\section{Conclusion}

The prevalence of carbapenemase producing bacilli has been increasing in our setting which worries microbiologists as well as clinicians to prescribing carbapenems. NDM was found to be the most prevalent carbapenemase genes among clinical isolates and belong to Indian lineage. That we need for implementation of drastic infection control measures and regular surveillance to prevent further spread of these resistant organisms among the hospital isolates. In addition,screening for carbapenemase production should be performed in any Gram-negative isolates with any slight decrease in susceptibility to carbapenems.

\section{Declarations}

\section{Acknowledgments}

We would like to thank the medical and technical staff of Medical Microbiology Department in Soba University Hospital, University of Khartoum for their help in strain and data collection.

\section{Funding}

This research received partial research fund from the Ministry of Higher Education and Scientific Research, Sudan (grant number SUD/MOH/MMAH/08/2016). 


\section{Authors' contributions}

$H E, M A$ and KE designed the study. HE carried out the microbiological and molecular analysis. HE and HA analysed the data. HE and MA wrote the first draft, KE, EM, and HA were contributors in revising the manuscript. All authors read and approved the final manuscript

\section{Ethics approval}

Formal permission was obtained from the managers of Soba University Hospital and the Institutional Research Ethics Committee of the Institute of Endemic Diseases, University of Khartoum, approved this study under reference number IEND_REC 12/2017. Patient consent was waived by the Research Ethics Committee.

\section{Data availability}

Data is available upon request from the first author.

\section{Conflict of interests}

All the authors declare on conflicts of interests.

\section{Author details}

${ }^{1}$ Soba University Hospital, Khartoum, Sudan

2 Institute of Endemic Diseases, University of Khartoum, Khartoum, Sudan

${ }^{3}$ Ahfad University for Women, Omdurman

${ }^{4}$ Department of Microbiology, Faculty of Medicine, University of Khartoum, Khartoum, Sudan

${ }^{5}$ Department of Microbiology, Faculty of Medical Laboratory Sciences, Sudan for Sciences and Technology, Khartoum, Sudan

\section{References}

1. Hall BG, Barlow M. Evolution of the serine $\beta$-lactamases: past, present and future. Drug Resist Updat. 2007;7:111-23.

2. Zowawi H, Balkhy H, Walsh T, Paterson D. $\beta$-Lactamase production in key gram-negative pathogen isolates from the Arabian Peninsula. Clin Microbiol Rev. 2013;26(3):361-380. 
3. Codjoe FS, Donkor ES. Carbapenem Resistance: A Review. Med Sci. 2017;6(1):1.

4. Nordmann P, Naas T, Poirel L. Global Spread of Carbapenemase- producing Enterobacteriaceae. Emerg Infect Dis. 2011;17(10):1791-8.

5. Queenan AM, Bush K. Carbapenemases: the Versatile B-Lactamases. Clin Microbiol Rev. 2007;20(3):440-58.

6. Nordmann P, Gniadkowski M, Giske CG, Poirel L, Woodford N, Miriagou V. Identification and screening of carbapenemase-producing Enterobacteriaceae. Clin Microbiol Infect. 2012;18:432-8.

7. Moolchandani K, Sastry AS, Deepashree R, Sistla S, Harish BN, Mandal J. Antimicrobial Resistance Surveillance among Intensive Care Units of a Tertiary Care Hospital in Southern India. J Clin Diagn Res. 2017;11(2):1-7.

8. Wauters G, Vaneechoutte M. Approaches to the Identification of Aerobic Gram-Negative Bacteria. In: Manual of Clinical Microbiology. 11th ed. Washington, DC; 2015. p. 613-34.

9. CLSI. M100 Performance Standards for Antimicrobial. 27th ed. 2017. 106-143p.

10. Tsakris A, Kristo I, Poulou A, Themeli-digalaki K, Ikonomidis A, Petropoulou D, et al. Evaluation of boronic acid disk tests for differentiating KPC-Possessing Klebsiella pneumoniae isolates in the clinical laboratory. J Clin Microbiol. 2009;47(2):362-367.

11. Atschul S, Madden T, Schaffer A, et al. Gapped BLAST and PSI-BLAST. A new generation of protein database search programmes". NucleicAcid Res. 1997;25:3389-3402.

12. Kumar S, Stecher G, Tamura K. Molecular Evolutionary Genetics Analysis. Molecular Biology and Evolution; 2016. p. 1870-4.

13. Huang TD, Bogaerts P, Berhin C, Hoebeke M, Bauraing C, Glupczynski Y. Increasing proportion of carbapenemase-producing Enterobacteriaceae and emergence of a MCR-1 producer through a multicentric study among hospital-based and private laboratories in Belgium from September to November 2015. Eurosurveillance. 2017;1-9.

14. Pitout JDD, Nordmann P. Carbapenemase-Producing Klebsiella pneumoniae, a Key Pathogen Set for Global Nosocomial Dominance. Anti- microb Agents Chemother. 2015;59(10):5873-84.

15. Dahab RA, Ibrahim AM, Altayb HN. Phenotypic and genotypic detection of carbapenemase enzymes producing gram-negative bacilli isolated from patients in Khartoum State [version 1; referees: awaiting peer review]. F1000Research. 2017,6:1656

16. Ali MA. the prevalence and characterization of antibiotic resistance among Gram-negative bacilli. University of Khartoum; 2013.

17. Amer WH, Khalil HS, Abd ELWahab MAA. characterization of carpabenem resistant Enterobacteriaceae in Risk factors, phenotypic and genotypic characterization of carbapenem resistant Enterobacteriaceae in Tanta University Hospitals, Egypt. Int J Infect Control. 2016;12:1-11.

18. Okoche D, Asiimwe BB, Katabazi FA, Kato L. Prevalence and Characterization of CarbapenemResistant Enterobacteriaceae Isolated from Mulago National Referral. PLOS. 2015;48:1-11. 
19. Mushi MF, Mshana SE, Imirzalioglu C, Bwanga F. Carbapenemase Genes among Multidrug Resistant Gram Negative Clinical Isolates from a Tertiary Hospital in Mwanza, Tanzania. Biomed Res Int. 2014,3-8.

20. Perovic O, Micro FSA, Micro M, Britz E, Chb MB, Chetty V. IN PRACTICE Molecular detection of carbapenemase-producing genes in referral Enterobacteriaceae in South Africa : A short report. SAMJ. 2016;106(10):975-7.

21. Yusuf I, Arzai AH, Haruna M, Sharif AA, Getso MI. Detection of multi drug resistant bacteria in major hospitals in Kano,. Brazilian J Microbiol. 2014;45(3):791-8.

22. Mukonzo J, Namuwenge P, Okure G, Mwesige B, Namusisi O, Mukanga D. Over the counter suboptimal dispensing of antibiotics in Uganda. J Multidiscip Heal. 2013;6:303-310.

23. Karabay $O$, Altindis M, Koroglu M, Karatuna O, Aydemir ÖA. The carbapenem - resistant Enterobacteriaceae threat is growing: NDM - 1 epidemic at a training hospital in Turkey. Ann Clin Microbiol Antimicrob. 2016;15:1-6.

24. Deshpande P, Rodrigues C, Shetty A, Kapadia F, Hedge A, Soman R. New Delhi Metallo-beta lactamase (NDM-1) in Enterobacteriaceae: Treatment options with carbapenems compromised. J Assoc Physicians India. 2010;58:147-9.

25. Kumarasamy K, Toleman M, Walsh T, Al. E. Emergence of a new antibiotic resistance mechanism in India, Pakistan, and the UK: A molecular, biological, and epidemiological study. Lancet Infect Dis. 2010;10:597-602.

26. Sonnevend Á, Ghazawi A, Hashmey R, Jamal W, Rotimi V, Shibl A, et al. Characterization of carbapenem-resistant Enterobacteriaceae with high rate of autochtho- nous transmission in the arabian peninsula. PLoS One. 2015;10:e0131372.

27. Gülmez D, Woodford N, Palepou M-F, Mushtaq S, Metan G, Yakupogullari Y, et al. Carbapenemresistant Escherichia coli and Klebsiella pneumoniae isolates from Turkey with OXA-48-like carbapenemases and outer membrane protein loss. Int J Antimicrob Agents. 2008,31:523-6.

28. Memish Z, Assiri A, Almasri M, Al. E. Molecular characterization of carbapenemase production among Gram-negative bacteria in Saudi Arabia. Microb Drug Resist. 2015;21(3):307-314.

29. Giske CG. Global dissemination of extensively drug-resistant carbapenemase-producing Enterobacteriaceae: clinical perspectives on detection, treatment and infection control. J Intern Med. 2014,501-12.

30. Bonnin A, Nordmann P. First Identification of Novel NDM Carbapenemase, NDM- 7, in Escherichia coli in France. 2013;8(4):1-5.

31. Doi Y, Paterson DL. Carbapenemase-Producing Enterobacteriaceae. Semin Respir Crit Care Med. 2015;36:74-84.

32. Zowawi HM, Sartor AL, Sidjabat HE, Balkhy HH, Walsh TR, Johani M Al. Molecular Epidemiology of Carbapenem-Resistant Acinetobacter baumannii Isolates in the Gulf Cooperation Council States: Dominance of OXA-23-Type Producers. JClinical Microbiol. 2015;53(3):896-903. 
33. Karaaslan A, Soysal A, Gelmez GA, Kadayifci EK. Molecular characterization and risk factors for carbapenem-resistant Gram-negative bacilli colonization in children : emergence of NDM-producing Acinetobacter baumannii in a newborn intensive care unit in Turkey. J Hosp Infect. 2016;92:67-72.

34. Vanegas JM, Parra OL, Jiménez JN. Molecular epidemiology of carbapenem resistant gram-negative bacilli from infected pediatric population in tertiary - care hospitals in Medellín, Colombia: an increasing problem. BMC Infect Dis. 2016;1-10.

35. Bhaskar M, Anand R, Harish B. Prevalence of blaNDM-1 producing blood isolates of Escherichia coli, Klebsiella species and Enterobacter species in a tertiary care centre in South India. J Microbiol Res Rev. 2013;1(6):61-68.

36. Lin MY, Lyles-Banks RD, Lolans $K$ et al. The importance of long-term acute care hospitals in the regional epidemiology of Klebsiella pneumoniae carbapenemase-producing Enterobacteriaceae. Clin Infect Dis. 2013;57:1246-52.

37. Yusuf I, Magashi A, Firdausi F, Al. E. Phenotypic detection of car-bapenemase in members of Enterobacteriaceae in Kano, Nigeria. Int J Sci Technol. 2012;2(11):802-80.

38. Levy HG, Gould I, Endimiani A, Al. E. Detection, treatment, and prevention of carbapenemaseproducing Enterobacteriaceae: recom- mendations from an International Working Group. $J$ Chemother. 2013;25(3):129-140.

39. Legese M hailu, Weldearegay gebru M, Asrat D. Extended-spectrum beta-lactamase-and among Ethiopian children. Infect Drug Resist. 2017;10:27-34.

40. Nordmann P, Poirel L, Toleman MA, Walsh T. "Does broad-spectrum B-lactam resistance due to NDM-1 herald the end of the antibiotic era for treatment of infections caused by Gram-negative bacteria?" J Antimicrob Chemother. 2011;66(4):689-692.

41. Zhang $X$, Chen $D, X u G$, Huang $W$, Wang $X$. Molecular epidemiology and drug resistant mechanism in carbapenem-resistant Klebsiella pneumoniae isolated from pediatric patients in Shanghai, China. PLoS One. 2018;20:1-10.

42. Nithya N, Remitha R, Jayasree PR, Faisal M, Manish Kumar PR. Analysis of beta-lactamases, blaNDM-1phylogeny \&amp; plasmid replicons in multidrug-resistant Klebsiella spp. from a tertiary care centre in south India. Indian J Med Res. $2017 \mathrm{Jul} ; 146$ (Supplement):S38-45.

43. Cohen S, Leverstein-Van J, Hall M. Dutch Working Party on the Detection of Highly Resistant Microorganisms. Guideline for pheno- typic screening and confirmation of carbapenemases in Enterobacte- riaceae. Int J Antimicrob Agents. 2010;36(3):205-210.

44. Srinivasan R, Karaoz U, Volegova M, MacKichan J, Kato-Maeda M, Miller S, et al. Use of 16S rRNA gene for identification of a broad range of clinically relevant bacterial pathogens. PLoS One. 2015;10(2):e0117617.

45. Liu Y, Liu C, Zheng W, Zhang X, Yu J, Gao Q, et al. PCR detection of Klebsiella pneumoniae in infant formula based on 16S-23S internal transcribed spacer. Int J Food Microbiol. 2008;125(3):230-5.

46. Sabat G, Rose P, Hickey WJ, Harkin JM. Selective and sensitive method for PCR amplification of Escherichia coli 16S rRNA genes in soil. Appl Environ Microbiol. 2000;66(2):844-9. 
47. Spilker T, Coenye T, Vandamme P, Lipuma JJ. PCR-Based Assay for Differentiation of Pseudomonas aeruginosa from Other Pseudomonas Species Recovered from Cystic Fibrosis Patients Theodore. ASM. 2004;42(5):2074-9.

48. Higgins $P G$, Wisplinghoff $H$, Krut $O$, Seifert H. A PCR-based method to differentiate between Acinetobacter baumannii and Acinetobacter genomic species 13TU. CMI. 2007;13:1199-1201.

49. Hong D, Bae I, Jang I, Jeong S, Kang H, Lee K. Epidemiology and Characteristics of Metallo- $\beta$ Lactamase-Producing Pseudomonas aeruginosa. Infect Chemother. 2015;42(2):81-97.

50. Suwantarat N, Carroll KC. Epidemiology and molecular characterization of multidrug-resistant Gramnegative bacteria in Southeast Asia. Antimicrob Resist Infect Control. 2016;1-8.

51. Pasteran F, Mendez T, Guerriero L, Rapoport M, Corso A. Sensitive Screening Tests for Suspected Class A Carbapenemase Production in Species of Enterobacteriaceae. J Clin Microbiol. 2009;47(6):1631-9.

52. Lartigue M, Poirel L, Decousser J, Nordmann P. Multidrug-resistant Shigella sonnei and Salmonella enterica serotype Typhimurium isolates producing CTXMbeta- lactamases as causes of communityacquired infection in France. Clin Infect Dis. 2005;40:1069-1070.

53. Isturiz RE CC. Antibiotic use in developing countries. Infect Control Hosp Epidemiol. 2000;21(6):3947.

\section{Tables}

Due to technical limitations, Tables 1 - 5 are only available for download from the Supplementary Files section.

\section{Figures}




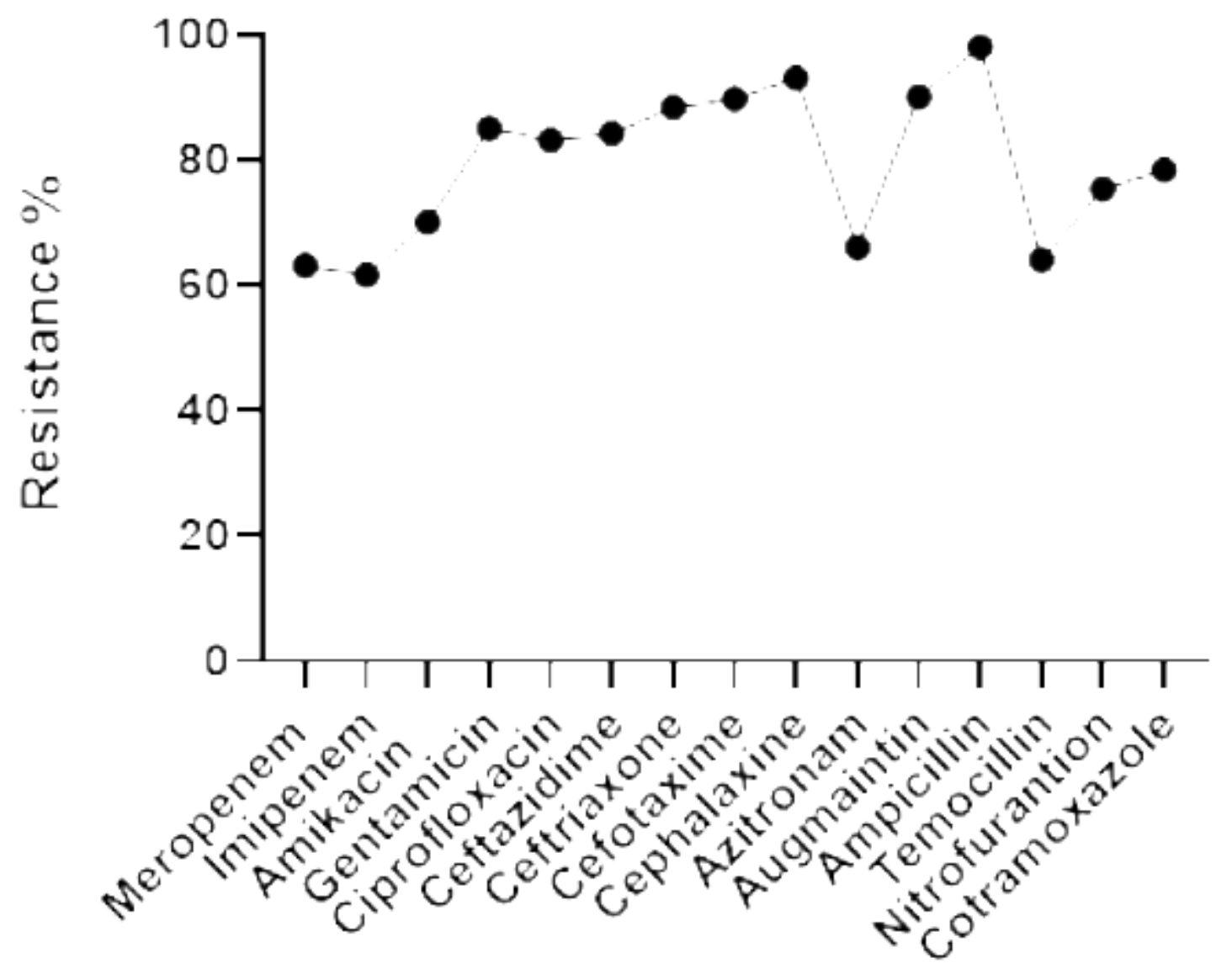

Antibiotic

Figure 1

Antimicrobial Resistance pattern among different Gram-negative bacilli isolated from patients treated at Khartoum state hospitals. 


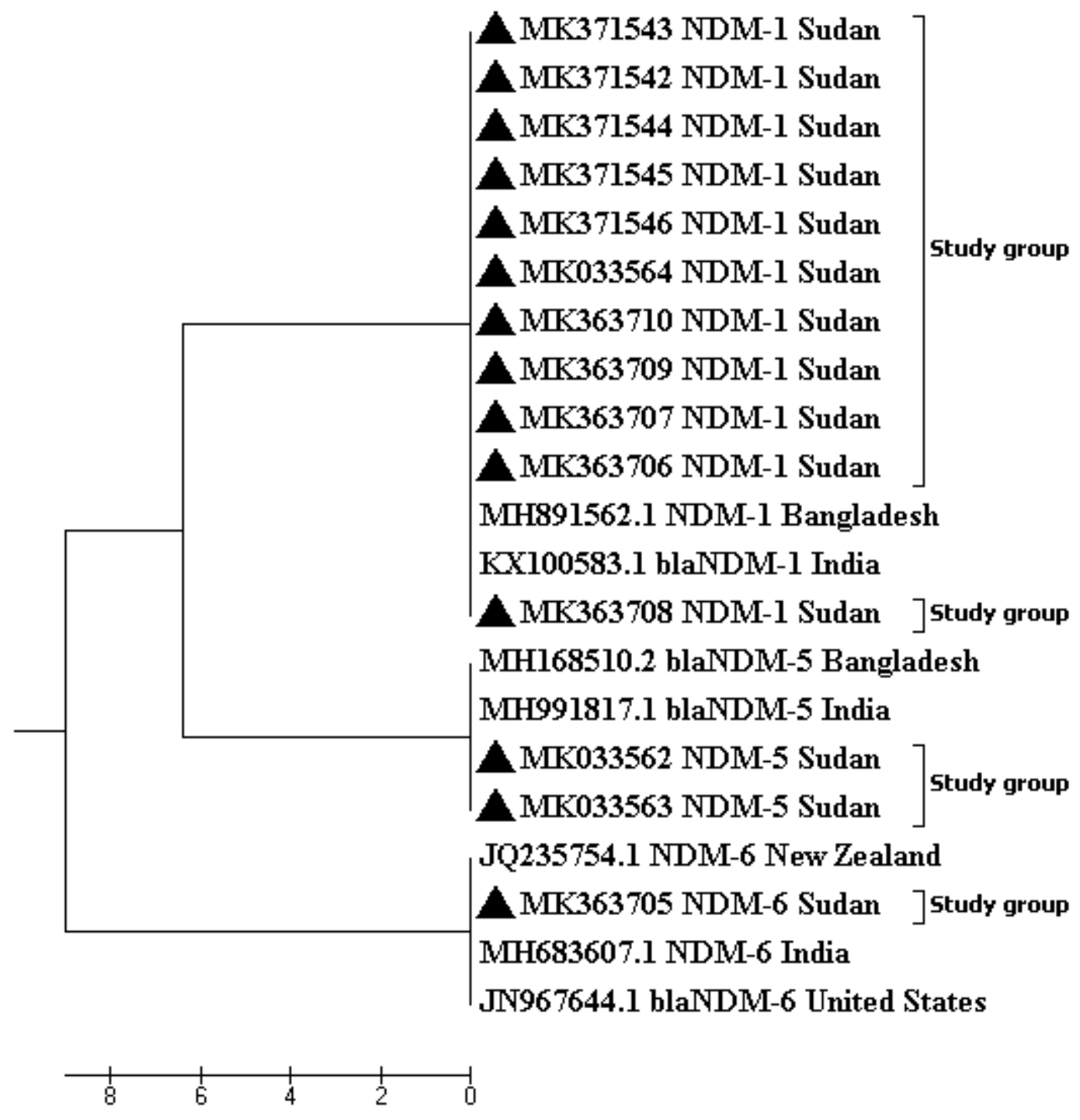

Figure 2

Phylogenetic tree of the 14 NDM isolated from different Gram-negative bacilli: Phylogenetic tree of the 14 NDM genes, Sequences were analysed using MEGA7, the neighbor-joining method, and bootstrap analysis (1,000 replicates) based on the ClustalW algorithm. The scale bar indicates 0.1 nucleotide substitutions per site. Reference sequences shown as: accession number, gene subtype, country. Sequences isolated in this study are designated by grey triangle.

\section{Supplementary Files}

This is a list of supplementary files associated with this preprint. Click to download.

- Table2.pdf

- Table4.pdf

- Table5.pdf 
- Table1.pdf

- Table3.pdf 